LA-UR-02-2041

\title{
Parton model versus color dipole formulation of the Drell-Yan process
}

\author{
Jörg Raufeisen $\left.\right|^{a}$, Jen-Chieh Peng ${ }^{a, b}$, and Gouranga C. Nayak ${ }^{a}$ \\ ${ }^{a}$ Los Alamos National Laboratory, Los Alamos, New Mexico 87545, USA \\ ${ }^{b}$ Department of Physics, University of Illinois, Urbana, Illinois 61801, USA
}

\begin{abstract}
In the kinematical region where the center of mass energy is much larger than all other scales, the Drell-Yan process can be formulated in the target rest frame in terms of the same color dipole cross section as low Bjorken- $x$ deep inelastic scattering. Since the mechanisms for heavy dilepton production appear very different in the dipole approach and in the conventional parton model, one may wonder whether these two formulations really represent the same physics. We perform a comparison of numerical calculations in the color dipole approach with calculations in the next-to-leading order parton model. For proton-proton scattering, the results are very similar at low $x_{2}$ from fixed target to RHIC energies, confirming the close connection between these two very different approaches. We also compare the transverse momentum distributions of Drell-Yan dileptons predicted in both formulations. The range of applicability of the dipole formulation and the impact of future Drell-Yan data from RHIC for determining the color dipole cross section are discussed. A detailed derivation of the dipole formulation of the Drell-Yan process is also included.
\end{abstract}

PACS: 13.85.Qk; 13.85.Lg; 13.60.Hb

Keywords: Drell-Yan process; dipole cross section; perturbative QCD

\footnotetext{
${ }^{1}$ email: jorgr@lanl.gov
} 


\section{Introduction}

With the advent of RHIC, the Drell-Yan(DY) process [1] can be studied in a new kinematical regime, the so-called Regge regime, where the dilepton mass $M$ is small compared to the $\mathrm{cm}$ energy $\sqrt{s}$, but still much larger than $\Lambda_{Q C D}$. The DY process at RHIC and LHC energies is therefore of similar interest as DIS at HERA, where one can study $\gamma^{*}$-proton scattering in the Regge regime. The new experimental possibilities motivate further theoretical investigations of the DY process. First of all, one needs a framework to calculate nuclear shadowing in the DY process, the onset of which can already be observed at fixed-target energy [2]. This is especially important in view of the RHIC heavy ion program. The color dipole formulation of the DY process introduced in [3, 4] is suitable to address this issue and a considerable amount of work in this direction has been performed [5]-97. In addition, the low- $x_{2}$ DY cross sections are sensitive to integrals over the color dipole cross section which are not accessible in DIS [4]. Therefore, future DY data can be used to further constrain this quantity. Most importantly, while the dipole approach has already been used in sophisticated analyses like the extraction of energy loss from E866/NuSea Drell-Yan data [8], its validity for protonproton interactions has never been established. This will be remedied with the present work.

In this paper we compare next-to-leading order (NLO) parton model calculations for DY dilepton production in proton-proton $(p p)$ and proton-deuteron $(p d)$ collisions with calculations in the dipole approach over a wide energy range. Although the two approaches are believed to be equivalent in a certain kinematical range, the underlying mechanisms appear to be quite different, and there is no known way to prove this equivalence analytically. However, both approaches are supposed to describe the same process, so they should yield similar numerical resultst.

Before we compare the results of the numerical calculations, the key features of the two approaches are briefly summarized. The well known mechanism for continuum dilepton production, which was first found more than thirty years ago by Drell and Yan [1], was formulated in a frame where both colliding hadrons are fast moving (infinite momentum frame). According to Feynman's picture of high energy collisions, the colliding objects can be viewed as collections of noninteracting partons with negligibly small transverse momenta. To lowest order, DY dileptons are produced by quark-antiquark annihilation, and the cross section reads,

$$
\frac{d^{2} \sigma_{D Y}}{d M^{2} d x_{F}}=\frac{4 \pi \alpha_{e m}^{2}}{9 M^{2} s} \frac{1}{x_{1}+x_{2}} \sum_{f=1}^{N_{f}} Z_{f}^{2}\left[q_{f}\left(x_{1}\right) \bar{q}_{f}\left(x_{2}\right)+q_{f}\left(x_{2}\right) \bar{q}_{f}\left(x_{1}\right)\right] .
$$

The distribution function of a quark (antiquark) of flavor $f$ of the target or the projectile is denoted by $q_{f}\left(\bar{q}_{f}\right), N_{f}$ is the number of active flavors and $Z_{f}$ is the quark charge. The

\footnotetext{
${ }^{2}$ Differences between parton model and dipole approach are seen in the angular distribution of DY pairs, if saturation effects are included in the dipole cross section [10]. When we talk about equivalence of parton model and dipole approach, we mean equivalence up to higher twist effects, which are neglected in the parton model, but (at least partially) included in the phenomenological parameterization of the dipole cross section we employ.
} 
longitudinal momentum fractions of the projectile (target) parton, $x_{1}\left(x_{2}\right)$, can be expressed in terms of Lorentz invariant scalar products as

$$
x_{1}=\frac{2 P_{2} \cdot q}{s} \quad ; \quad x_{2}=\frac{2 P_{1} \cdot q}{s}
$$

where $P_{1}^{\mu}\left(P_{2}^{\mu}\right)$ is the projectile (target) four momentum, $q^{\mu}$ is the four momentum of the dilepton, $q^{2}=M^{2}>0$, and $x_{F}$ is the Feynman- $x, x_{F}=x_{1}-x_{2}$.

For most qualitative descriptions, it is sufficient to consider the DY process in terms of the lowest order annihilation process, Eq. (11). Calculations with Eq. (11), however, underestimate measured DY cross sections by an overall factor. It is necessary to employ the NLO framework for the DY process, in order to make quantitative predictions, see [1] for a review. In addition, the DY cross section differential in the dileptons transverse momentum receives huge corrections from higher order processes. Indeed, to lowest order, one would not expect dileptons with large transverse momentum $q_{\perp}$, in contrast to what is observed in experiment. Even though the occurrence of perturbatively large transverse momenta can be explained in NLO, it is not straightforward to calculate the shape of the $q_{\perp}$-distribution in the parton model. A resummation of large logarithms in $q_{\perp} / M$ [12] or alternatively the introduction of an intrinsic transverse momentum [13] is necessary to avoid the divergence of the differential cross section at $q_{\perp}=0$.

In the parton model, all nonperturbative effects are parameterized in the parton distribution functions $q_{f}, \bar{q}_{f}$, which evolve according to the DGLAP evolution equations. For DY in nuclear collisions, the parton distribution functions of the proton are simply replaced by empirical nuclear parton distribution functions [14. This approach does not explain the dynamical origin of the nuclear effects
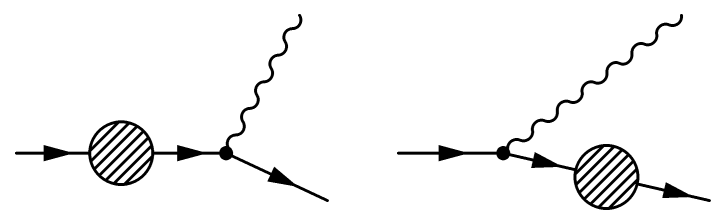

Figure 1: In the target rest frame, DY dilepton production looks like bremsstrahlung. A quark or an antiquark from the projectile hadron scatters off the target color field (denoted by the shaded circles) and radiates a massive photon, which subsequently decays into the lepton pair. The photon decay is not shown. The photon can be radiated before or after the quark (antiquark) scatters.

Nuclear effects, effects from higher orders in perturbation theory, as well as other possible nonperturbative effects, are more readily treated when the Drell-Yan process is viewed in the target rest frame. Note that although cross sections are Lorentz invariant, the partonic interpretation of high energy scattering processes does depend on the reference frame. In the rest frame of the target, the production mechanism for high mass continuum dileptons looks 
like bremsstrahlung [3, 4, see Fig. 1. In the high energy limit, when one can neglect terms that are suppressed by a factor 1/energy, each of the two graphs factorizes into a production vertex for the virtual photon times an amplitude for scattering a quark off the target. These scattering amplitudes combine in the squared matrix element in exactly the same way as in DIS, which makes it possible to express the DY cross section in terms of the same cross section $\sigma_{q \bar{q}}^{N}$ for scattering a $q \bar{q}$-dipole off a nucleon $(N)$ as in low- $x_{B j}$ DIS,

$$
\frac{d \sigma\left(q N \rightarrow \gamma^{*} X\right)}{d \ln \alpha}=\int d^{2} \rho\left|\Psi_{\gamma^{*} q}(\alpha, \rho)\right|^{2} \sigma_{q \bar{q}}^{N}(\alpha \rho, x)
$$

Here, $\alpha$ is the light-cone momentum fraction the virtual photon takes away from its parent quark, and $\rho$ is the transverse separation between $\gamma^{*}$ and final quark. The electromagnetic radiation, $q \rightarrow \gamma^{*} q$, is described by the light-cone wavefunction $\Psi_{\gamma^{*} q}(\alpha, \rho)$, see Eqs. (A.18) - (A.20), which can be calculated perturbatively. Summation over photon polarizations is understood in Eq. (3). The dipole cross section $\sigma_{q \bar{q}}^{N}$ is of nonperturbative origin and has to be taken from phenomenology. The energy scale $x$ of the dipole cross section will be discussed in the next section. A detailed derivation of Eq. (3) is given in the appendix.

Using a phenomenological parameterization for the dipole cross section in Eq. (3) is a very economical way to account for higher order and nonperturbative effects. The dipole approach can even be applied at low values of $M$ where perturbative QCD is not valid [15]. It was found in a recent analysis [10 that most of E772 DY data (except some points at low $M$ ) are reasonably well described in the dipole approach without introducing an arbitrary overall normalization factor. In addition it was found that the transverse momentum distribution does not diverge at $q_{\perp}=0$, even without intrinsic transverse momentum.

We emphasize that the dipole approach does not describe an additional production mechanism for heavy dileptons. Rather, the two approaches are believed to describe the same physics in different reference frames. Therefore, calculations in the NLO parton model and in the dipole approach should give similar results for the DY cross section. This is what we numerically check in this paper. In the following section, we compare numerical calculations of the DY cross section (integrated over the transverse momentum of the dilepton) in both approaches. In section 3, we also compare the predictions of dipole approach and parton model for the DY transverse momentum distribution at RHIC.

\section{Numerical comparison of the two approaches}

In order to perform calculations that can be compared with experimental data, one has to embed the partonic cross section, Eq. (3), into the hadronic environment. In the infinite momentum frame, the momentum fraction of the projectile quark is $x_{1}$, see Eq. (2). However, when the scalar product defining $x_{1}$ is evaluated in the target rest frame, one finds $x_{1}=\alpha z$, where $z=x_{1} / \alpha$ is the momentum fraction of the incoming proton carried by the projectile quark. The different meanings of $x_{1}$ in the target rest frame and in the infinite momentum frame is a manifestation of the frame dependence of partonic mechanisms. In the target rest frame, $x_{1}$ is the momentum fraction that the lepton pair takes from the projectile proton. 
Thus, one obtains for the proton-nucleon DY cross section

$$
\begin{aligned}
\frac{d^{2} \sigma\left(p N \rightarrow l^{+} l^{-} X\right)}{d M^{2} d x_{F}} & =\frac{\alpha_{e m}}{3 \pi M^{2}} \frac{x_{1}}{x_{1}+x_{2}} \int_{x_{1}}^{1} \frac{d \alpha}{\alpha^{2}} \sum_{f=1}^{N_{f}} Z_{f}^{2}\left[q_{f}\left(\frac{x_{1}}{\alpha}, \widetilde{Q}\right)+\bar{q}_{f}\left(\frac{x_{1}}{\alpha}, \widetilde{Q}\right)\right] \\
& \times \int d^{2} \rho\left|\Psi_{\gamma^{*} q}(\alpha, \rho)\right|^{2} \sigma_{q \bar{q}}^{N}(\alpha \rho, x) .
\end{aligned}
$$

We still need to know the scale $\widetilde{Q}$ at which the projectile parton distributions are probed and the energy $x$ at which the dipole cross section enters. These quantities are not known exactly, instead we have to rely on plausible arguments to determine their values. In order to find $\widetilde{Q}$, note that the transverse distances $\rho$ that contribute to the DY cross section are controlled by the extension parameter

$$
\eta^{2}=(1-\alpha) M^{2}+\alpha^{2} m_{f}^{2}
$$

The numerically dominant term in the LC wavefunctions, Eqs. (A.18, A.19), is the one that contains the Bessel function $\mathrm{K}_{1}(\eta \rho)$. Since this function decays exponentially at large arguments, the largest distances that can numerically contribute are of order $\sim 1 / \eta$. For fluctuations with $\alpha \rightarrow 1$, these distances can become of the order of a typical hadronic radius, in analogy to the aligned jet configurations in DIS [16]. On the other hand, the minimal value of $\alpha$ is $x_{1}$, so that the largest virtuality entering the calculation is $\widetilde{Q}^{2}=\eta_{\max }^{2}=\left(1-x_{1}\right) M^{2}$. We choose this quantity to be the hard scale at which the projectile parton distribution is probed. The parton distribution functions (PDFs) are taken from CERNLIB [17]. The quark mass is set to $m_{f}=0$ in all our calculations, see [10 for its numerical influence.

For the quark density of the projectile, we employ the leading order parameterization that corresponds to the NLO parameterization used in the parton model calculation. This means e.g. we use CTEQ5L in the dipole approach when comparing it with a NLO parton model calculation using CTEQ5M. One should use leading order PDFs in the dipole approach, because they are scheme independent and have a probabilistic interpretation.

The energy scale $x$ of the dipole cross section in Eq. (41) is determined from the analogy to DIS. In DIS, the argument of the dipole cross section is $x_{B j}=Q^{2} / W^{2}$, where $Q$ is the virtuality of the photon and $W$ is the $\gamma^{*}$-proton $\mathrm{cm}$ energy. Therefore, we choose $x=M^{2} / \hat{s}=\alpha x_{2}$, where $\hat{s}=s x_{1} / \alpha$ is the quark-proton $\mathrm{cm}$ energy squared.

Note that in the previous analysis [10], $M^{2}$ and $x_{2}$, instead of $\widetilde{Q}^{2}$ and $x$, were used. The different choice of scales in this paper has the effect of increasing the cross section by a factor of up to 2 for dilepton mass $M \sim 4 \mathrm{GeV}$. This is mostly due to the different choice of $\widetilde{Q}^{2}$. Using $\alpha x_{2}$ instead of $x_{2}$ is only a $\sim 10 \%$ effect at $x_{2}<0.1$. These uncertainties vanish at larger masses, $M \sim 8 \mathrm{GeV}$. 


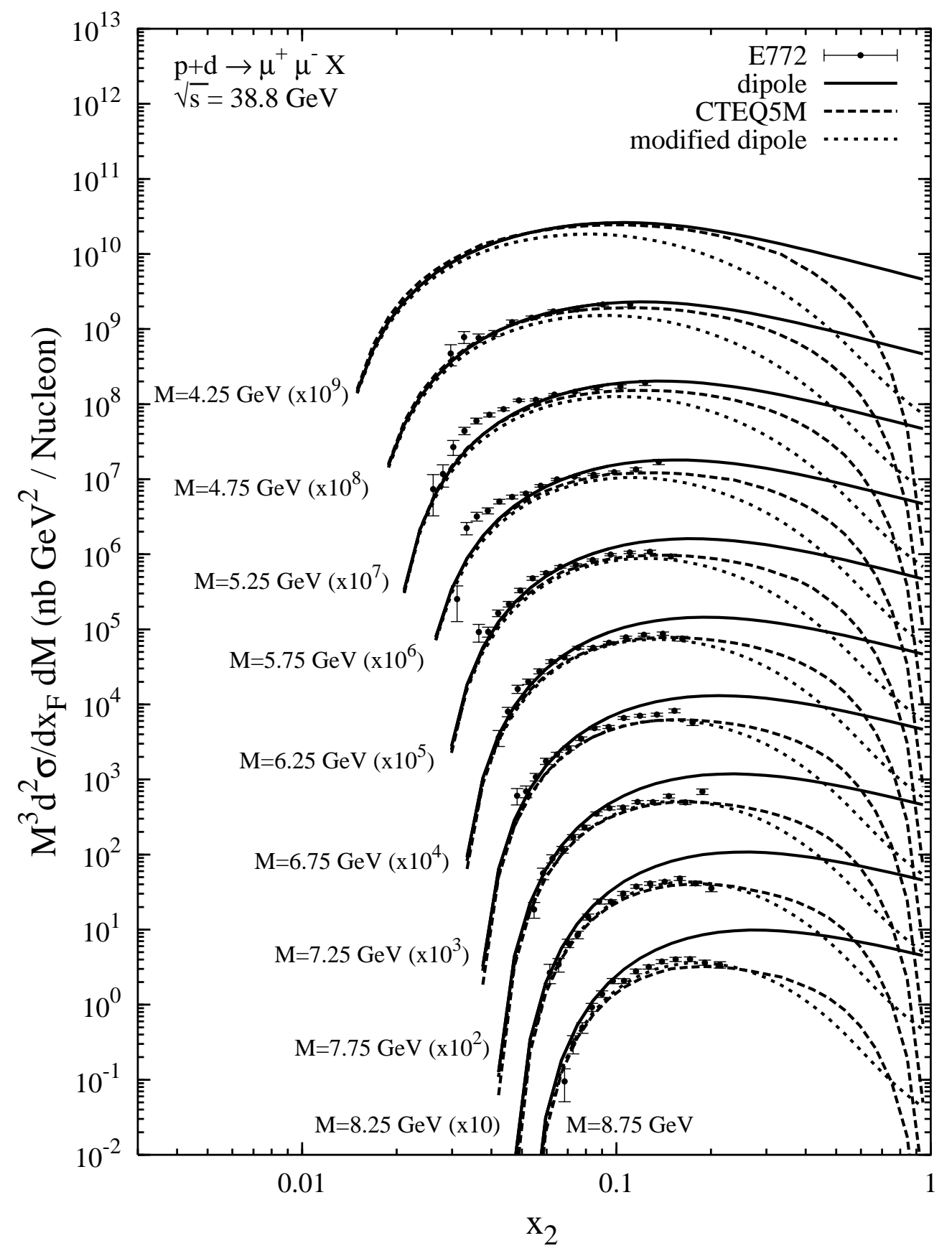

Figure 2: Calculations in the dipole approach to DY and its modification Eq. (9) compared to NLO parton model results at fixed target energy $(\sqrt{s}=38.8 \mathrm{GeV})$. The CTEQ5M parameterization [29] is used for the parton model calculation. The data are from E772 [23]. The curves and data for the different mass bins have been rescaled for better visibility. In all calculations, none of the free parameters of the dipole approach were adjusted to DY data. Only DIS data have been used. 
For the calculations in the dipole approach shown in Figs. 2 - 4, we employ the parameterization of the color dipole cross section by Golec-Biernat and Wüsthoff [18,

$$
\sigma_{q \bar{q}}^{N}(\rho, x)=\sigma_{0}\left[1-\exp \left(-\frac{\rho^{2} Q_{s}^{2}(x)}{4}\right)\right],
$$

with $\sigma_{0}=23.03 \mathrm{mb}$. This parameterization rises quadratically at small separations $\rho$ as demanded by color transparency [19] and saturates at large separations. The saturation scale that controls the flattening of the dipole cross section is given by

$$
Q_{s}^{2}(x)=1 \mathrm{GeV}^{2}\left(\frac{0.0003}{x}\right)^{0.288} .
$$

We point out that no DY data have been used to determine the parameters in Eq. (6). Only DIS data from HERA were fitted to extract the dipole cross section.

Note that the parameterization Eq. (6) contains only the Pomeron part of the dipole cross section. As a consequence, the dipole approach predicts identical cross sections for particle and antiparticle induced DY. This is, of course, reproduced in the parton model at low $x_{2}$. In addition to this Pomeron part, there is also a contribution from Reggeon exchange, corresponding to valence quarks in the target [9]. In principle, one could fit a parameterization of this Reggeon part to the data, but the predictive power of such a procedure would be very low, since the Reggeon part, in contrast to the Pomeron part, depends on the colliding hadrons. We therefore do not attempt to determine the Reggeon contribution to the dipole cross section in this paper.

The NLO parton model calculation was performed with the CTEQ code [20] provided earlier to the E866/Nusea collaboration by W.K. Tung [21]. We use the dilepton mass $M$ as hard scale in the strong coupling constant and in the parton distributions. This is the standard choice. The code assumes $\overline{M S}$ renormalization scheme.

Fig. 2 shows calculations at fixed target energy in the dipole approach and in the NLO parton model, as well as experimental DY data from E772 [23]. The lowest value of $x_{2}$ that can be reached is about 0.02 . Dipole and parton model calculations are very similar at low $x_{2}$, where the dipole approach is supposed to be valid, but strongly deviate as $x_{2} \rightarrow 1$. Clearly, we cannot apply the dipole approach at large $x_{2}$, since it does not include valence quark contributions to the DY cross section (see [10] for a more detailed discussion of effects that are taken into account by the dipole approach). Note also that the parameterization Eq. (6) was fitted only to DIS data below $x_{B j}<0.01$, hence the curves shown in Fig. 2 are already an extrapolation of the fit.

In Fig. 2 there are several E772 data points at low $x_{2}$ that exceed the calculations in both the parton model and dipole approaches. This excess was also visible for some points in [10]. Indeed, experiment E772 was not designed to measure the absolute normalization of the DY cross section, but to measure nuclear effects. New data from E866/NuSea will not suffer from such problems. A comparison between preliminary E866/NuSea data and the NLO parton model in [25] shows good agreement.

The disagreement between the two approaches sets in around $x_{2} \sim 0.1$ in Fig. 2. Somewhat surprisingly, the dipole approach yields values that exceed the prediction from the 
parton model (at large $x_{2}$ ), even though the dipole approach does not include several contributions to the DY cross section. This can be understood, if one observes that the dipole cross section is related to the target gluon density $G$. At small separations $\rho$ one has 26.

$$
\sigma_{q \bar{q}}^{N}(\rho, x)=\frac{\pi^{2}}{3} \alpha_{s}\left(\frac{\lambda}{\rho^{2}}\right) \rho^{2} x G\left(x, \frac{\lambda}{\rho^{2}}\right) .
$$

Eqs. (6) and (7) contain only the part of the gluon density that increases at low $x$. At large $x$, however, the gluon density decreases like $G(x) \propto(1-x)^{5}$ [27]. At high virtuality, the power is even higher than 5 , say $\sim 8$. In order to estimate the uncertainty originating from this effect, we follow [28] and replace the saturation scale Eq. (7) by

$$
Q_{s}^{2}(x) \rightarrow Q_{s}^{2}(x)(1-x)^{5} .
$$

We refer to this recipe as modified dipole approach and the numerical results are shown by the short dashed curves in Figs. 2- - . The replacement Eq. (9) has virtually no influence at $x<0.01$, where the parameters in Eq. (6) have been fitted in [18], but at $x=0.1$, it reduces the cross section by almost a factor of 2 . In fact, in [29] calculations in the dipole approach were performed employing a dipole cross section calculated from a gluon density that contains saturation effects. For $\sqrt{s}=38.8 \mathrm{GeV}$, the results of [29] lie below the ones obtained in [10] with the saturation model of Golec-Biernat and Wüsthoff [18] by a factor of $\sim 2$. We believe this disagreement is largely due to the decreasing gluon density at $x_{2}>0.01$, i.e. due to uncertainties in the dipole cross section.

Apparently, the low- $x_{2}$ range accessible at fixed target energies is very limited, and therefore the dipole formulation is bestowed with several uncertainties. Unfortunately, there are no DY data at really low $x_{2}$. Even CDF data [30] are at about the same values of $x_{2}$ as the E772 data, because they are mostly in the $Z$-boson mass range. At RHIC, however, much lower values of $x_{2}$ will be reached, where the dipole approach can readily be applied. In Fig. 3, we show predictions from the dipole approach and the NLO parton model (using CTEQ5M PDFs) for RHIC energy. Calculations with the modified dipole cross section, Eq. (9), are also shown. The disagreement between dipole approach and parton model sets in around $x_{2} \sim 0.01$, i.e. around $x_{F} \sim 0$. At low $x_{2}$ all three curves are in good agreement, confirming the similarity between the two approaches.

In order to show the uncertainty arising from the choice of PDFs, we did the same calculation, employing GRV98HO [24], see Fig. 困. In this case, the differences between the two approaches are slightly larger than for CTEQ5M. With GRV98HO, the NLO parton model calculation exceeds the dipole approach by at most a factor of 1.5 for $x_{2}<0.01$; for CTEQ5M, the corresponding value is only about 1.2. The third standard PDF we employed was MRST(c-g) [31. The results are comparable to GRV98HO and are not shown here, because they do not provide any further insight. For most values of $x_{2}$ and $M$, which do not lie at the very edge of the phase space, the NLO calculations are only $\sim 10 \%-20 \%$ higher than the dipole approach. In summary, we find that the uncertainty from the choice of PDFs is $\sim 25 \%$ at the low values of $x_{2}$ shown in Figs. 3 and 4 . 


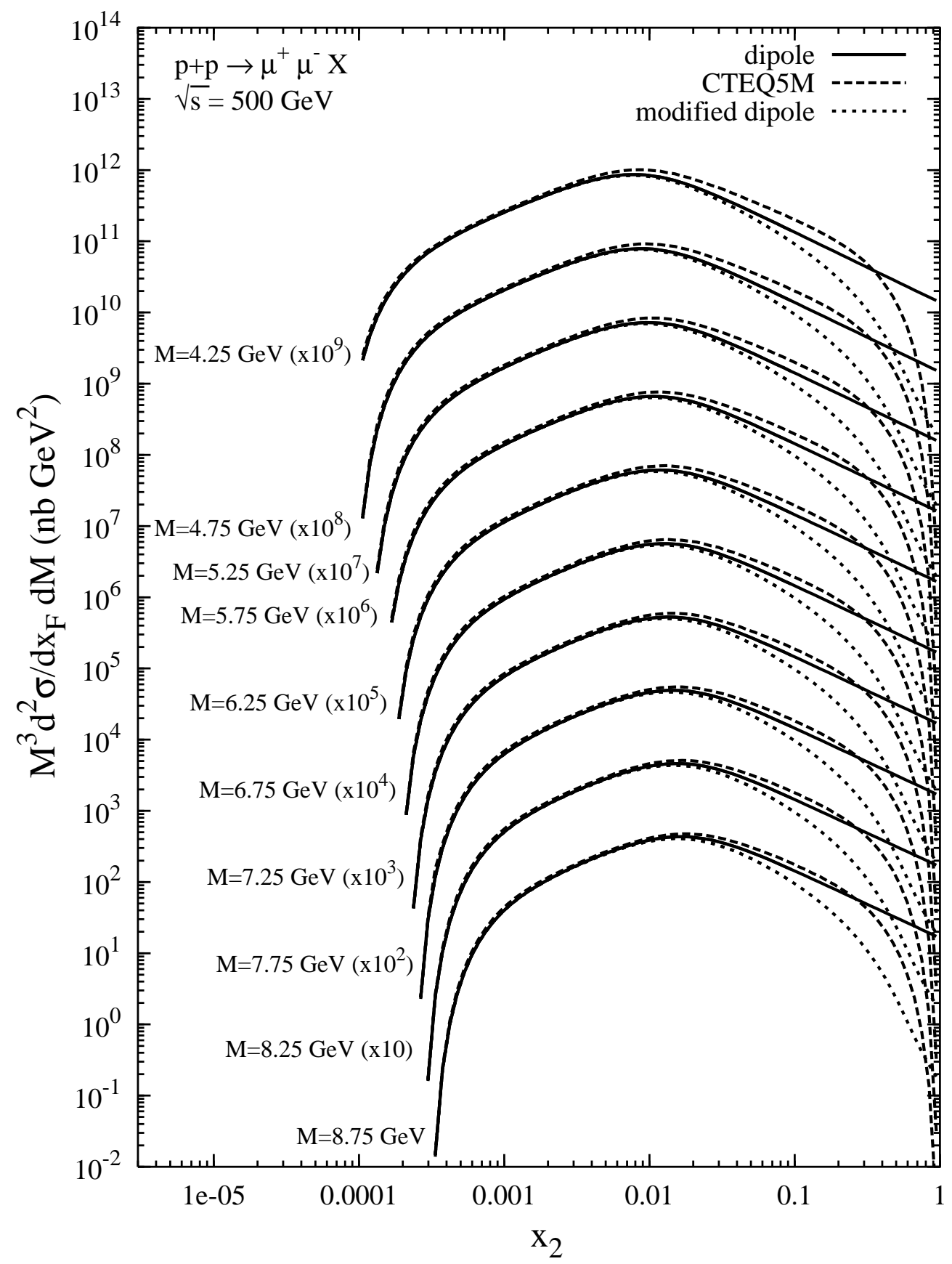

Figure 3: The same as Fig. 圆, but now at RHIC energy $(\sqrt{s}=500$ $\mathrm{GeV})$, where much lower values of $x_{2}$ can be reached. 


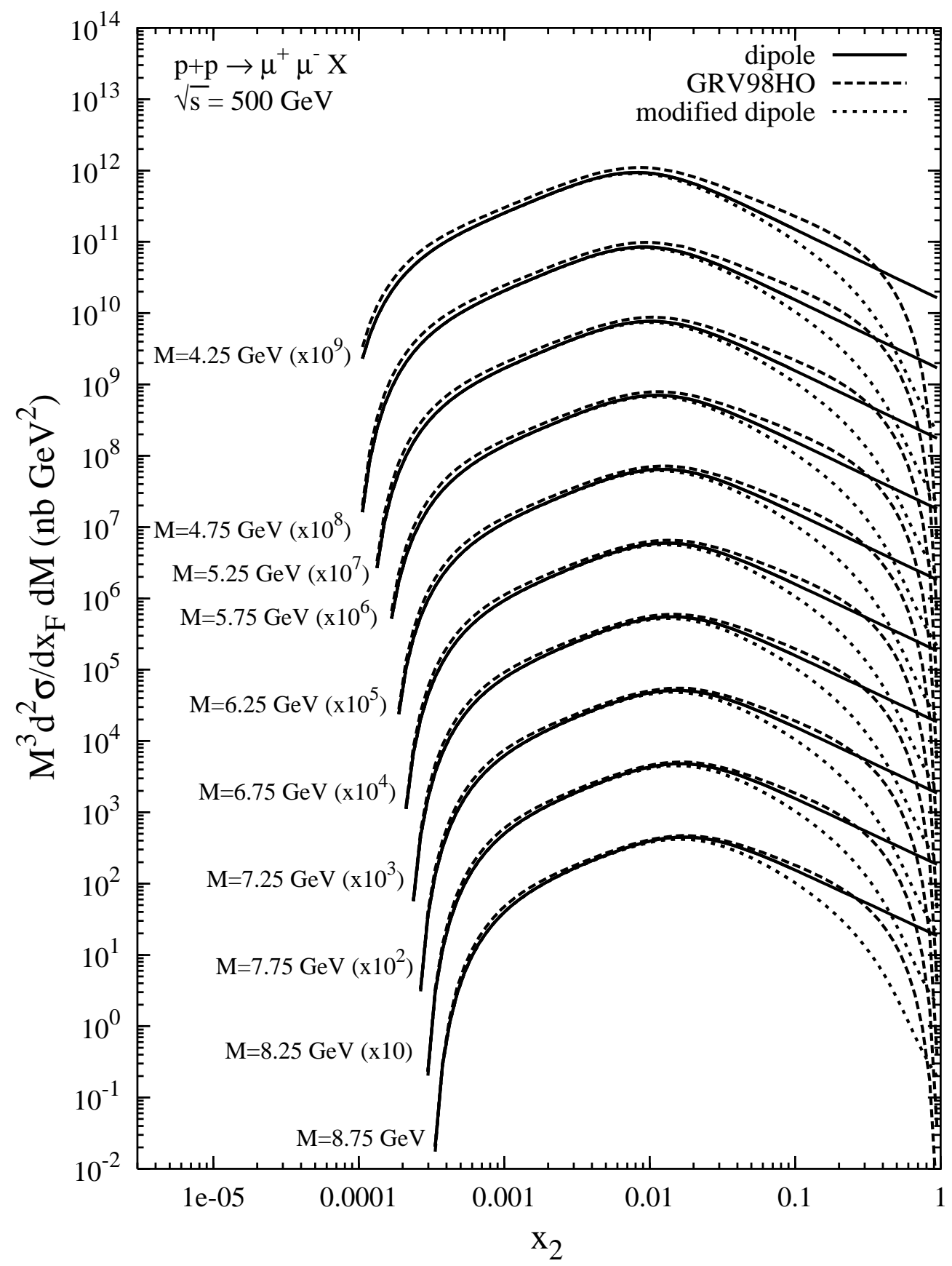

Figure 4: The same as Fig. 园, but with GRV PDFs [24]. For the dipole approach, we employed the LO PDFs GRV98LO for the projectile parton distribution. 
We also point out that for these low values of $x_{2}$, the DY $K$-factor is typically 1.4 to 1.5 and can become even smaller than unity for very low $x_{2}$. In the parton model, the $K$-factor is defined as the DY cross section calculated in NLO divided by the same quantity calculated in LO. The $K$-factor originates (mostly) from the analytic continuation from the spacelike (DIS) to the timelike (DY) region of $q^{2}$ in the annihilation and virtual corrections at NLO. The QCD Compton process, which dominates at low $x_{2}$ because of the large target gluon density, does (almost) not contribute to the $K$-factor. Therefore, in the kinematical region of interest, the $K$-factor is considerably below its usually assumed value of 2 to 3 .

At this point, we stress that the term " $K$-factor" is defined only in the parton model. In the dipole approach, there is no freedom to adjust the overall normalization of the DY cross section as proposed in [29], because higher order corrections are contained in the parameterization of the dipole cross section.

Most uncertainties in the dipole approach arise from uncertainties in this parameterization. It remains to be seen whether future low $x_{2}$ DY data from RHIC can be accurately described with a (probably improved) phenomenological parameterization of the dipole cross section. We believe that future DY data can serve as an important source of knowledge about the color dipole cross section.

\section{The DY transverse momentum distribution}

The DY cross section differential in the dilepton transverse momentum $q_{\perp}$ can provide even more detailed information about the shape of the dipole cross section than the $q_{\perp}$-integrated cross section. In the dipole approach, the $q_{\perp}$-differential cross section reads [5],

$$
\begin{aligned}
\frac{d^{3} \sigma\left(q p \rightarrow \gamma^{*} X\right)}{d \ln \alpha d^{2} q_{\perp}} & =\frac{1}{(2 \pi)^{2}} \int d^{2} \rho_{1} d^{2} \rho_{2} \mathrm{e}^{\mathrm{i} \vec{q}_{\perp} \cdot\left(\vec{\rho}_{1}-\vec{\rho}_{2}\right)} \Psi_{\gamma^{*} q}^{*}\left(\alpha, \vec{\rho}_{1}\right) \Psi_{\gamma^{*} q}\left(\alpha, \vec{\rho}_{2}\right) \\
& \times \frac{1}{2}\left\{\sigma_{q \bar{q}}\left(\alpha \rho_{1}, x\right)+\sigma_{q \bar{q}}\left(\alpha \rho_{2}, x\right)-\sigma_{q \bar{q}}\left(\alpha\left(\vec{\rho}_{1}-\vec{\rho}_{2}\right), x\right)\right\}
\end{aligned}
$$

where $q_{\perp}$ is the $\gamma^{*}$ transverse momentum wrt the direction of the projectile quark. This formula was first published in [5]. We give a detailed derivation in the appendix. The partonic cross section, Eq. (10), has to be embedded into the hadronic environment in the same way as in Eq. (田). Again, the sum over photon polarizations is understood to be contained in the light-cone wavefunctions, see Eq. (A.20).

We would like to compare the transverse momentum distribution calculated in the dipole approach with the same quantity computed in the parton model. Since the CTEQ code used in the previous section is not capable of calculating the DY transverse momentum distribution, we developed our own code based on the formulas given in [13]. Again, we use the dilepton mass $M$ as hard scale. It was already mentioned in the introduction, that there are no large transverse momenta in the leading order parton model. The order $\alpha_{s}$ correction yields a transverse momentum distribution that diverges at $q_{\perp} \rightarrow 0$ and has the wrong shape, when compared to data [13]. This can be remedied by resumming contributions from soft gluon radiation [12], which yields a good description of the data. 
In this paper, however, we follow [13 and apply a more phenomenological recipe. We introduce a soft, nonperturbative, primordial transverse momentum distribution of the partons in the colliding protons, which we parameterize by a Gaussian,

$$
f\left(k_{\perp}^{2}\right)=\frac{1}{4 \pi \sigma_{q}^{2}} \mathrm{e}^{\frac{-k_{\perp}^{2}}{4 \sigma_{q}^{2}}} .
$$

The mean transverse primordial momentum squared of a single constituent in the proton is then

$$
\left\langle k_{\perp}^{2}\right\rangle_{\text {primordial }}=2 \sigma_{q}^{2}
$$

The perturbatively calculated $q_{\perp}$-distribution is then smeared out by this primordial momentum and the regularized dilepton cross section is given by

$$
\begin{aligned}
\left(\frac{d^{4} \sigma^{D Y}}{d M^{2} d x_{F} d^{2} q_{\perp}}\right)_{\mathrm{reg}} & =\int d^{2} p_{\perp}\left(\frac{d^{4} \sigma^{D Y}}{d M^{2} d x_{F} d^{2} p_{\perp}}\right)_{\mathrm{A}+\mathrm{C}}\left[f\left(\left(\vec{q}_{\perp}-\vec{p}_{\perp}\right)^{2}\right)-f\left(q_{\perp}^{2}\right)\right] \\
& +f\left(q_{\perp}^{2}\right)\left(\frac{d^{2} \sigma^{D Y}}{d M^{2} d x_{F}}\right)_{\mathrm{NLO}+\mathrm{LO}} .
\end{aligned}
$$

The subscript $A+C$ refers to "annihilation+Compton" correction. The virtual corrections do not contribute to the first term in Eq. (13). With this recipe, the perturbative result is reproduced for large transverse momenta while the divergence at $q_{\perp}=0$ is removed. The first term on the rhs of Eq. (13) vanished after integration over $q_{\perp}$ and $\int d^{2} q_{\perp} f\left(q_{\perp}\right)$ is normalized to unity, so that the total DY cross section is correctly reproduced.

The results of our calculations in the dipole approach and in the parton model are shown in Fig. 5. We performed the parton model calculation for two different values of the smearing parameter $\sigma_{q}$. The lower value, $\sigma_{q}=0.48 \mathrm{GeV}$, was used in [32] to describe ISR data at $\sqrt{s}=44 \mathrm{GeV}$ and $62 \mathrm{GeV}$. The parameter $\sigma_{q}$ is presumably independent of energy $s$. Nevertheless, we also calculated for twice the ISR value of $\sigma_{q}$, because at the low values of $x$ reached at RHIC, quarks may have a larger intrinsic transverse momentum. Of course, the choice of $\sigma_{q}$ has the largest influence at small $q_{\perp}$, while at very large transverse momentum, the results become independent of this parameter. Note however, that $\sigma_{q}$ still has a rather large impact on the numerical results at intermediate $q_{\perp} \sim 2-3 \mathrm{GeV}$.

No intrinsic transverse momentum is included in the dipole approach, even though one could introduce one. As already pointed out in [10], the cross section calculated in the dipole approach does not diverge at $q_{\perp} \rightarrow 0$ because of the flattening of the dipole cross section, see Eq. (6). At $q_{\perp}=10 \mathrm{GeV}$, dipole approach and parton model differ by about a factor of 5 . Employing the modified dipole approach, Eq. (9), brings the curve down by a factor of 0.9 at $q_{\perp}=10 \mathrm{GeV}$ and has no effect at small $q_{\perp}$. Therefore, we do not show the corresponding curves. Note that at high transverse momentum, the numerical value of the DY cross section is completely insensitive to the large $\rho$ behavior of the dipole cross section. Indeed, it was found in [10] that an expansion of Eq. (6) to order $\rho^{2}$ virtually yields the same numerical results at large $q_{\perp}$ when employed in Eq. (10). On the other hand, the behavior at $q_{\perp} \rightarrow 0$ is largely determined by the large $\rho$ behavior of the dipole cross section. Therefore, future data on the DY $q_{\perp}$-distribution can be important for further constraining the dipole cross section, provided statistics will be high enough. 


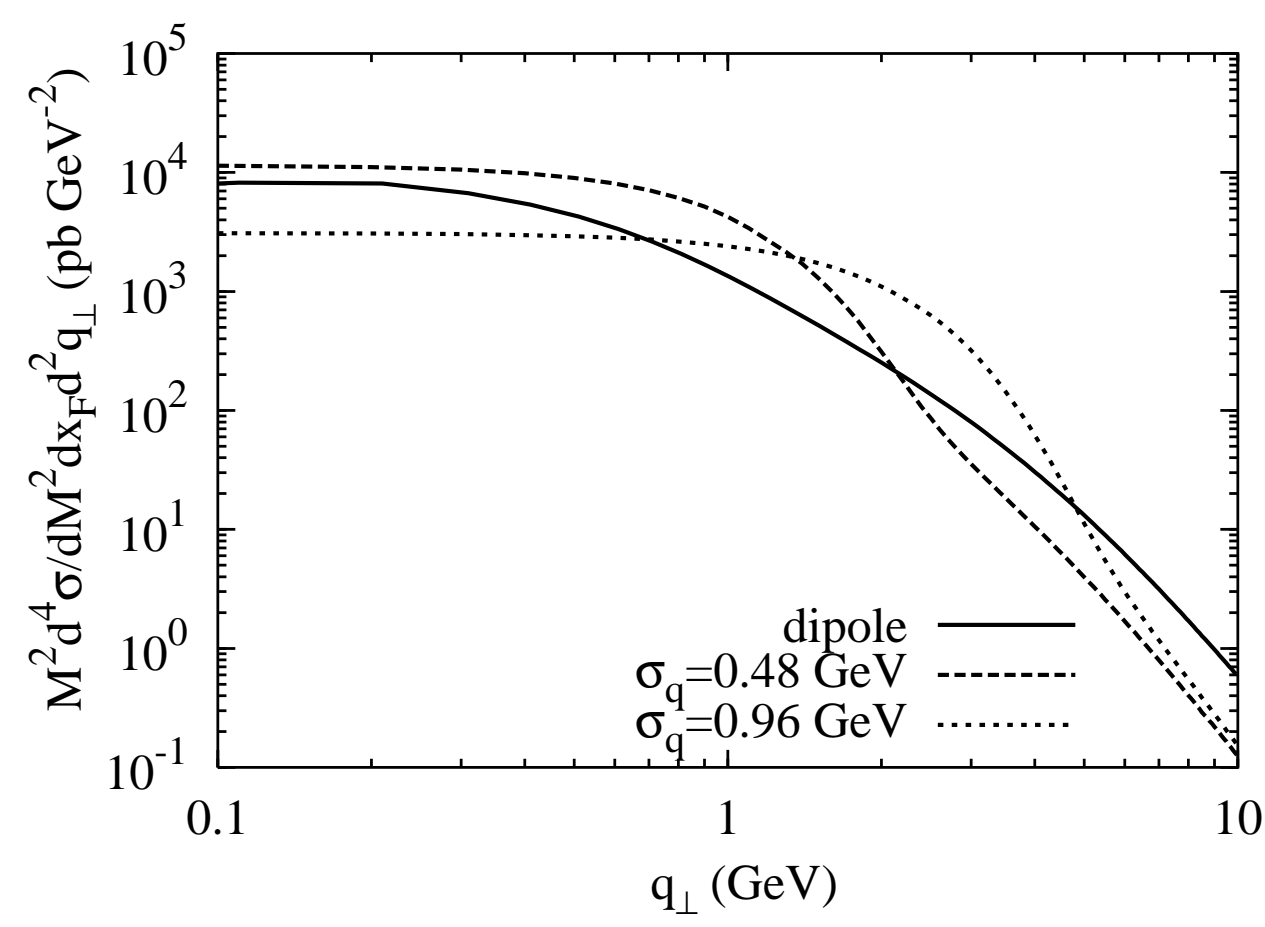

Figure 5: Comparison of the DY transverse momentum distribution calculated in the dipole approach and in the NLO parton model. The parton model calculations have been performed for two different values of the quark's intrinsic momentum. The values of the kinematical variables for all curves in this figure are $\sqrt{s}=500 \mathrm{GeV}, x_{F}=0$ and $M=4.25$ $\mathrm{GeV}$.

\section{Summary}

In this paper, we presented a detailed comparison between calculations of the Drell-Yan cross section in the color dipole approach with calculations in the conventional NLO parton model. We also compared both approaches with experimental data when available. The dipole approach and the parton model are believed to represent the same physics, but viewed from different reference frames. The purpose of this paper was to demonstrate the close connection between the two approaches numerically.

First, we compared calculations for the DY cross section integrated over the dilepton's transverse momentum $q_{\perp}$ and found that at low $x_{2}$, where the dipole approach is supposed to be valid, calculations agree well. Since both approaches rely on different nonperturbative inputs, numerical results will not agree exactly. There are several uncertainties from free parameters that can affect the numerical value of the Drell-Yan cross section. In the dipole approach, these uncertainties are:

- Different possible choices of the hard scale $\widetilde{Q}$, at which the parton distribution of the projecetile is probed, can lead to an uncertainty as large as a factor of 2 for $M \sim 4 \mathrm{GeV}$, 
even though the dependence on $\widetilde{Q}$ is only logarithmic. This uncertainty vanishes with increasing dilepton mass $M$. For $M=8.75 \mathrm{GeV}$ it is only $\sim 10 \%$. Note that a larger value of $\widetilde{Q}$ leads to a smaller value of the DY cross section, because the projectile PDF is probed at large momentum fraction.

- For the low values of $x_{2}$ we are interested in, different parameterizations of PDFs lead to variations of the numerical results of up to $\sim 25 \%$. This uncertainty is also present in the parton model.

- The uncertainty arising from possible choices of the energy scale $x$ is $\sim 10 \%$. Since the dipole cross section increases with decreasing $x$, a lower value of $x$ pulls the DY cross section up.

- The dipole approach allows one to introduce a quark mass $m_{f}$. In this work we set $m_{q}=0$. A quark mass $m_{q}=200 \mathrm{MeV}$ would reduce the numerical value of the DY cross section by less than $10 \%$ [10].

The main uncertainty, which is difficult to quantify, arises from the parameterization of the dipole cross section. Future DY data from RHIC will certainly help to better constrain this quantity.

We found that the disagreement between the two approaches is typically of order $20 \%$ for $x_{2}<0.01$. We believe that the parameterization of the dipole cross section can be adjusted so that future RHIC data can be described without introducing an arbitrary overall normalization factor for which we cannot find a theoretical justification. For larger values of $x_{2}$, the applicability of the dipole approach is questionable, because this approach neglects several contribution to the DY cross section which might already be important for $0.01 \leq$ $x_{2} \leq 0.1$.

Note that several data points from E772 23] at low $x_{2}$ are not well described by neither of the approaches. Preliminary E866/NuSea data [25], however, which were measured in the same kinematical region, are well described by the NLO parton model. Therefore, the agreement between dipole approach and NLO parton model at low $x_{2}$ gives confidence into the applicability of the dipole approach. However, we stress that in order to strictly prove the validity of the dipole approach, one has to reproduce experimental data and not the parton model calculation.

Naturally, the differences between the two approaches are larger when one considers the DY transverse momentum distribution. At low $q_{\perp}$, the numerical result in the parton model depends quite strongly on the amount of primordial transverse momentum which is included. This uncertainty is however no longer present at very large transverse momenta, $q_{\perp} \sim 10 \mathrm{GeV}$, where the two calculations differ by a factor of 5 (at $\sqrt{s}=500 \mathrm{GeV}$ ). It will be seen, if RHIC data can distinguish between the two approaches or if the dipole cross section and/or the parton distribution function can both be adjusted to fit the data. We point out that both approaches predict the same asymptotic behavior for the partonic DY cross section, namely $d \sigma / d^{2} q_{\perp} \propto 1 / q_{\perp}^{4}$ for large transverse momentum [5]. 
Acknowledgments: We are indebted to Fred Cooper, Mikkel Johnson, Boris Kopeliovich and Magno Machado for valuable discussion. This work was supported by the U.S. Department of Energy at Los Alamos National Laboratory under Contract No. W7405-ENG-38.

\section{Appendix A Derivation of the dipole formula for DY}

In this appendix, we give a detailed derivation of the formula that expresses the DY cross section in terms of the color dipole cross section, Eq. (3). This formula was first discovered in [3]. Some details of the derivation in the semiclassical approach of [33] are given in the appendix of [4]. In this paper, we use the framework of covariant Feynman perturbation theory. The calculation can be carried out in a very similar way for DIS.

The differential cross section for dilepton production in the target rest frame, see Fig. 11, is given by

$$
\begin{aligned}
d^{8} \sigma\left(q N \rightarrow q l^{+} l^{-} X\right) & =\sum_{X} \sum_{\lambda \lambda^{\prime}} \epsilon_{\mu}^{*}(\lambda) \epsilon_{\nu}\left(\lambda^{\prime}\right) \overline{\mathcal{M}}^{\mu \nu} \frac{d \alpha d^{2} q_{\perp} d^{2} p_{f \perp}}{(2 \pi)^{5} 8\left(p_{i}^{0}\right)^{2} \alpha(1-\alpha)} \\
& \times \alpha_{e m} \epsilon_{\kappa}(\lambda) \epsilon_{\rho}^{*}\left(\lambda^{\prime}\right) L^{\rho \kappa} \frac{d M^{2} d \Omega}{16 \pi^{2} M^{4}}
\end{aligned}
$$

where $p_{i}$ is the momentum of the projectile quark, $p_{f}$ is the momentum of the final quark and $q$ is the momentum of the massive photon, $q^{2}=M^{2}>0$. The first line describes the bremsstrahlung of a massive photon from a quark (as depicted in Fig. 1), and the second line the decay into the lepton pair $l^{+} l^{-}$into solid angle $d \Omega$ (not shown in Fig. 四). The electromagnetic coupling constant is denoted by $\alpha_{e m}=e^{2} /(4 \pi)=1 / 137$. Furthermore, $\epsilon_{\mu}(\lambda)$ is the polarization vector of the photon, $\lambda \in\{ \pm 1,0\}$. We sum over all final states $X$ and the photon polarizations $\lambda, \lambda^{\prime}$. The $\delta$-function for energy conservation is already integrated over. Furthermore, $\alpha$ is the energy fraction the photon takes from its parent quark. It is assumed that only transverse momentum but no energy is exchanged with the target. Then, $\overline{\mathcal{M}}^{\mu \nu}$ is the absolute square of the bremsstrahlungs-amplitude, before contraction with the polarization vector of the photon. There are two contributions to this amplitude,

$$
\overline{\mathcal{M}}^{\mu \nu}=\frac{1}{2} \sum_{\sigma_{f} \sigma_{i}} \frac{1}{N_{c}} \sum_{c_{f} c_{i}}\left(\mathcal{M}_{s}^{\mu}+\mathcal{M}_{u}^{\mu}\right)\left(\mathcal{M}_{s}^{* \nu}+\mathcal{M}_{u}^{* \nu}\right) .
$$

The $s$-channel amplitude $\mathcal{M}_{s}^{\mu}$ describes the process where the photon is radiated after the quark has scattered off the target, Fig. 1 (left), and the $u$-channel amplitude $\mathcal{M}_{u}^{\mu}$ describes the process where the photon is radiated before the quark scatters off the proton, Fig. 11 (right). The bar indicates that the matrix element is summed over helicity $\sigma_{f}$ and color $c_{f}$ of the final quark and averaged over helicity $\sigma_{i}$ and color $c_{i}$ of the initial quark. $\left(N_{c}=3\right.$ is the number of colors.) Finally, $p_{l^{ \pm}}$are the dilepton momenta and

$$
L^{\mu \nu}=4\left(p_{l^{+}}^{\mu} p_{l^{-}}^{\nu}+p_{l^{+}}^{\nu} p_{l^{-}}^{\mu}-g^{\mu \nu} p_{l^{+}} p_{l^{-}}\right)
$$


is the leptonic tensor, summed over helicities.

Different terms in the sum over photon helicities lead to different angular distributions of the lepton pair. In this paper, we integrate over the solid angle $d \Omega=d \phi d(\cos \theta)$, and obtain for the differential DY cross section

$$
\frac{d^{4} \sigma\left(q N \rightarrow l^{+} l^{-} X\right)}{d \ln \alpha d M^{2} d^{2} q_{\perp}}=\frac{\alpha_{e m}}{3 \pi M^{2}}\left\{\frac{d^{3} \sigma_{T}\left(q N \rightarrow \gamma^{*} X\right)}{d \ln \alpha d^{2} q_{\perp}}+\frac{d^{3} \sigma_{L}\left(q N \rightarrow \gamma^{*} X\right)}{d \ln \alpha d^{2} q_{\perp}}\right\}
$$

where the indices $T$ and $L$ stand for transverse and longitudinal photons, respectively. We also integrated over the phase space of the final quark. The factor $\alpha_{e m} /\left(3 \pi M^{2}\right)$ originates from the second line of Eq. (A.1) and describes the decay of the $\gamma^{*}$ into the lepton pair. The interesting physics resides in the quantities $d^{3} \sigma_{T, L} / d \ln \alpha d^{2} q_{\perp}$, which are given by

$$
\begin{aligned}
& \frac{d^{3} \sigma_{T}\left(q N \rightarrow \gamma^{*} X\right)}{d \ln \alpha d^{2} q_{\perp}}=\int d^{2} p_{f \perp} \sum_{X} \sum_{\lambda \in\{ \pm 1\}} \frac{\epsilon_{\mu}^{*}(\lambda) \epsilon_{\nu}(\lambda) \overline{\mathcal{M}}^{\mu \nu}}{(2 \pi)^{5} 8\left(p_{i}^{0}\right)^{2}(1-\alpha)}, \\
& \frac{d^{3} \sigma_{T}\left(q N \rightarrow \gamma^{*} X\right)}{d \ln \alpha d^{2} q_{\perp}}=\int d^{2} p_{f \perp} \sum_{X} \frac{\epsilon_{\mu}^{*}(\lambda=0) \epsilon_{\nu}(\lambda=0) \overline{\mathcal{M}}^{\mu \nu}}{(2 \pi)^{5} 8\left(p_{i}^{0}\right)^{2}(1-\alpha)} .
\end{aligned}
$$

Terms with $\lambda \neq \lambda^{\prime}$ in Eq. (A.1) vanish after integration over the azimuthal angle $\phi$.

We will now express the $d^{3} \sigma_{T, L} / d \ln \alpha d^{2} q_{\perp}$ in terms of the $q \rightarrow \gamma^{*} q$ light-cone(LC) wavefunctions and the dipole cross section. Consider the $s$-channel graph in Fig. 1 (left) where the photon is radiated after the projectile quark has scattered off the target. The propagator of the quark in the intermediate state can be written as

$$
\frac{\not p_{f}+\not q+m_{f}}{\left(p_{f}+q\right)^{2}-m_{f}^{2}}=\sum_{\sigma} \frac{u_{\sigma}\left(p_{f}+q\right) \bar{u}_{\sigma}\left(p_{f}+q\right)}{\left(p_{f}+q\right)^{2}-m_{f}^{2}}-\frac{\gamma^{+}}{2\left(p_{f}^{+}+q^{+}\right)} .
$$

The $u_{\sigma}(p)$ are Dirac-spinors, $\left(\not p-m_{f}\right) u_{\sigma}(p)=0$, for on-shell momentum $p$ and helicity $\sigma$. The $\gamma^{+}$-term arises, because the intermediate quark is off-shell. In the high energy approximation which we employ, this term is dropped [3. This is the crucial step that allows one to write the amplitude as a product of an LC wavefunction and a quark scattering amplitude.

The $s$-channel amplitude then turns out to be

$$
\mathrm{i} \mathcal{M}_{s}^{\mu}=e \sum_{\sigma} \frac{\bar{u}_{\sigma_{f}}\left(p_{f}\right) \gamma^{\mu} u_{\sigma}\left(p_{f}+q\right)}{\left(p_{f}+q\right)^{2}-m_{f}^{2}} t_{q, \sigma \sigma_{i}}\left(\left(p_{f}^{0}+q^{0}\right), \vec{k}_{\perp}\right) .
$$

For simplicity, we have set the flavor charge to unity. Here, $t_{q, \sigma \sigma_{i}}\left(\left(p_{f}^{0}+q^{0}\right), \vec{k}_{\perp}\right)$ is the amplitude for scattering a quark off a nucleon in the rest frame of the nucleon,

$$
t_{q, \sigma \sigma_{i}}\left(\left(p_{f}^{0}+q^{0}\right), \vec{k}_{\perp}\right)=\bar{u}_{\sigma}\left(p_{f}+q\right) \gamma^{0} V_{q}\left(\vec{k}_{\perp}\right) u_{\sigma_{i}}\left(p_{i}\right) \approx 2 p_{i}^{0} \delta_{\sigma, \sigma_{i}} V_{q}\left(\vec{k}_{\perp}\right) .
$$

\footnotetext{
${ }^{3}$ As already pointed out in 国, this approximation is equivalent to neglecting the instantaneous vertices in a Hamiltonian light-cone approach to QCD [34], and violates gauge invariance. The color dipole approach is gauge invariant only in leading $\log (x)$ approximation.
} 
Note that $t$ and $V$ are matrices in color space, we suppressed the color indices. Our spinors are normalized to $u_{\sigma}^{\dagger}(p) u_{\sigma^{\prime}}(p)=2 p^{0} \delta_{\sigma, \sigma^{\prime}}$. The function $V_{q}\left(\vec{k}_{\perp}\right)$ will be completely absorbed into the dipole cross section, so that we will never need to specify it. Therefore, the dipole formulation also accounts for some higher order and nonperturbative effects, which are difficult or even impossible to account for in the standard parton model. We write $V_{q}$ only as function of the exchanged transverse momentum $\vec{k}_{\perp}=\vec{p}_{f \perp}+\vec{q}_{\perp}-\vec{p}_{i \perp}$, because the longitudinal momentum of the projectile quark cannot be changed significantly at high energies. Note that $V_{q}$ also depends on energy, even though we do not write this dependence explicitly. The Kronecker- $\delta_{\sigma, \sigma_{i}}$ means that the helicity of the quark is not changed by scattering off the proton.

In impact parameter space, the amplitude reads

$$
\begin{aligned}
\widetilde{\mathcal{M}}_{s}^{\mu}(\vec{b}, \vec{\rho}) & =\int \frac{d^{2} l_{\perp} d^{2} k_{\perp}}{(2 \pi)^{4}} \mathrm{e}^{-\mathrm{i} \vec{l}_{\perp} \cdot \alpha \vec{\rho}-\mathrm{i} \vec{k}_{\perp} \cdot \vec{b}} \mathcal{M}_{s}^{\mu}\left(\vec{l}_{\perp}, \vec{k}_{\perp}\right) \\
& =-\mathrm{i} \sqrt{4 \pi} \frac{\sqrt{1-\alpha}}{\alpha^{2}} \Psi_{\gamma^{*} q}^{\mu}(\alpha, \vec{\rho}) 2 p_{i}^{0} \widetilde{V}_{q}(\vec{b})
\end{aligned}
$$

where $\vec{l}_{\perp}=\vec{p}_{f \perp}-(1-\alpha) \vec{q}_{\perp} / \alpha$ is the transverse momentum of the final quark in a frame where the $z$-axis is parallel to the photon momentum. The conjugate variable to $\vec{l}_{\perp}$, namely $\alpha \vec{\rho}$, is then the transverse distance between initial and final quark in that frame. This quantity will become the distance variable the dipole cross section. Furthermore,

$$
\widetilde{V}_{q}(\vec{b})=\int \frac{d^{2} k_{\perp}}{(2 \pi)^{2}} \mathrm{e}^{-\mathrm{i} \vec{k}_{\perp} \cdot \vec{b}} V_{q}\left(\vec{k}_{\perp}\right)
$$

The LC wavefunctions in impact parameter space are related to the quark-photon vertex and the propagator by Fourier transformation,

$$
\Psi_{\gamma^{*} q}^{\mu}(\alpha, \vec{\rho})=\alpha^{3} \sqrt{1-\alpha} \int \frac{d^{2} l_{\perp}}{(2 \pi)^{2}} \mathrm{e}^{-\mathrm{i} \vec{l}_{\perp} \cdot \alpha \vec{\rho}} \sqrt{\alpha_{e m}} \frac{\bar{u}_{\sigma_{f}}\left(p_{f}\right) \gamma^{\mu} u_{\sigma_{i}}\left(p_{f}+q\right)}{\alpha^{2} l_{\perp}^{2}+\eta^{2}}
$$

where $\eta^{2}=(1-\alpha) M^{2}+\alpha^{2} m_{f}^{2}$. In our numerical calculations in this paper, we set the quark mass $m_{f}=0$. See [0] for its influence.

The $u$-channel graph can be written in the same way as (A.9), but with a quark scattering amplitude at a shifted impact parameter. This can be seen from the propagators. While the propagator for the $s$-channel graph yields

$$
\frac{1}{\left(p_{f}+q\right)^{2}-m_{f}^{2}}=\frac{\alpha(1-\alpha)}{\alpha^{2} l_{\perp}^{2}+\eta^{2}}
$$

one finds for the $u$-channel graph the combination $\vec{l}_{\perp}+\vec{k}_{\perp}$ instead of $l_{\perp}$,

$$
\frac{1}{\left(p_{i}-q\right)^{2}-m_{f}^{2}}=-\frac{\alpha}{\alpha^{2}\left(\vec{l}_{\perp}+\vec{k}_{\perp}\right)^{2}+\eta^{2}} .
$$


One then obtains for the $u$-channel amplitude in impact parameter space

$$
\begin{aligned}
\widetilde{\mathcal{M}}_{u}^{\mu}(\vec{b}, \vec{\rho}) & =\mathrm{i} \frac{\sqrt{4 \pi}}{\alpha^{2} \sqrt{1-\alpha}} \Psi_{\gamma^{*} q}^{\mu}(\alpha, \vec{\rho}) 2 p_{f}^{0} \widetilde{V}_{q}(\vec{b}+\alpha \vec{\rho}) \\
& =\mathrm{i} \sqrt{4 \pi} \frac{\sqrt{1-\alpha}}{\alpha^{2}} \Psi_{\gamma^{*} q}^{\mu}(\alpha, \vec{\rho}) 2 p_{i}^{0} \widetilde{V}_{q}(\vec{b}+\alpha \vec{\rho}) .
\end{aligned}
$$

and with Eqs. (A.4) and (A.5) for the photon production cross sections

$$
\begin{aligned}
\frac{d^{3} \sigma_{T, L}\left(q N \rightarrow \gamma^{*} X\right)}{d \ln \alpha d^{2} q_{\perp}} & =\int d^{2} p_{f \perp} \frac{1}{(2 \pi)^{4}} \int d^{2} b_{1} \int d^{2} b_{2} \int d^{2} \rho_{1} \int d^{2} \rho_{2} \\
& \times \mathrm{e}^{\mathrm{i} \vec{l}_{\perp} \cdot \alpha\left(\vec{\rho}_{1}-\vec{\rho}_{2}\right)+\mathrm{i} \vec{k}_{\perp} \cdot\left(\vec{b}_{1}-\vec{b}_{2}\right)} \sum_{\lambda} \frac{1}{2} \sum_{\sigma_{f} \sigma_{i}} \Psi_{\gamma^{*} q}^{\lambda}\left(\alpha, \vec{\rho}_{1}\right) \Psi_{\gamma^{*} q}^{\lambda *}\left(\alpha, \vec{\rho}_{2}\right) \\
& \times \sum_{X} \frac{1}{N_{c}} \sum_{c_{f} c_{i}}\left\{\widetilde{V}_{q}\left(\vec{b}_{1}\right)-\widetilde{V}_{q}\left(\vec{b}_{1}+\alpha \vec{\rho}_{1}\right)\right\}\left\{\widetilde{V}_{q}^{\dagger}\left(\vec{b}_{2}\right)-\widetilde{V}_{q}^{\dagger}\left(\vec{b}_{2}+\alpha \vec{\rho}_{2}\right)\right\}
\end{aligned}
$$

where $\vec{l}_{\perp}=\vec{p}_{f \perp}-(1-\alpha) \vec{q}_{\perp} / \alpha$ and $\vec{k}_{\perp}=\vec{p}_{f \perp}+\vec{q}_{\perp}-\vec{p}_{i \perp}$. In the transverse case $(T)$, the sum over $\lambda$ includes both transverse polarizations, $\lambda= \pm 1$, while in the longitudinal case $(L)$ only one term, $\lambda=0$ appears in the sum.

The LC wavefunctions for radiation of transverse and longitudinal photons are readily calculated by contracting Eq. (A.12) with the photon polarization vector [3, [4, (5],

$$
\begin{aligned}
\Psi_{\gamma^{*} q}^{T}\left(\alpha, \vec{\rho}_{1}\right) \Psi_{\gamma^{*} q}^{T *}\left(\alpha, \vec{\rho}_{2}\right) & =\sum_{\lambda= \pm 1} \frac{1}{2} \sum_{\sigma_{f} \sigma_{i}} \epsilon_{\mu}^{*}(\lambda) \Psi_{\gamma^{*} q}^{\mu}\left(\alpha, \vec{\rho}_{1}\right) \epsilon_{\mu}(\lambda) \Psi_{\gamma^{*} q}^{\mu *}\left(\alpha, \vec{\rho}_{2}\right) \\
& =\frac{\alpha_{e m}}{2 \pi^{2}}\left\{m_{f}^{2} \alpha^{4} \mathrm{~K}_{0}\left(\eta \rho_{1}\right) \mathrm{K}_{0}\left(\eta \rho_{2}\right)\right. \\
& \left.+\left[1+(1-\alpha)^{2}\right] \eta^{2} \frac{\vec{\rho}_{1} \cdot \vec{\rho}_{2}}{\rho_{1} \rho_{2}} \mathrm{~K}_{1}\left(\eta \rho_{1}\right) \mathrm{K}_{1}\left(\eta \rho_{2}\right)\right\}, \\
\Psi_{\gamma^{*} q}^{L}\left(\alpha, \vec{\rho}_{1}\right) \Psi_{\gamma^{*} q}^{L *}\left(\alpha, \vec{\rho}_{2}\right) & =\frac{1}{2} \sum_{\sigma_{f} \sigma_{i}} \epsilon_{\mu}^{*}(\lambda=0) \Psi_{\gamma^{*} q}^{\lambda=0}\left(\alpha, \vec{\rho}_{1}\right) \epsilon_{\mu}(\lambda=0) \Psi_{\gamma^{*} q}^{* \lambda=0}\left(\alpha, \vec{\rho}_{2}\right) \\
& =\frac{\alpha_{e m}}{\pi^{2}} M^{2}(1-\alpha)^{2} \mathrm{~K}_{0}\left(\eta \rho_{1}\right) \mathrm{K}_{0}\left(\eta \rho_{2}\right) .
\end{aligned}
$$

Often, one needs only the sum over all polarization states of the photon. We denote the corresponding quantity by

$$
\Psi_{\gamma^{*} q}\left(\alpha, \vec{\rho}_{1}\right) \Psi_{\gamma^{*} q}^{*}\left(\alpha, \vec{\rho}_{2}\right)=\Psi_{\gamma^{*} q}^{T}\left(\alpha, \vec{\rho}_{1}\right) \Psi_{\gamma^{*} q}^{T *}\left(\alpha, \vec{\rho}_{2}\right)+\Psi_{\gamma^{*} q}^{L}\left(\alpha, \vec{\rho}_{1}\right) \Psi_{\gamma^{*} q}^{L *}\left(\alpha, \vec{\rho}_{2}\right) .
$$

Integrating (A.17) over the transverse momentum $q_{\perp}$ of the photon yields

$$
\frac{d \sigma_{T, L}\left(q N \rightarrow \gamma^{*} X\right)}{d \ln \alpha}=\int d^{2} \rho\left|\Psi_{\gamma^{*} q}^{T, L}(\alpha, \vec{\rho})\right|^{2} \sigma_{q \bar{q}}^{N}(\alpha \rho),
$$


with the dipole cross section

$$
\sigma_{q \bar{q}}(\alpha \rho)=\sum_{X} \frac{1}{N_{c}} \sum_{c_{f} c_{i}} \int d^{2} b\left|\widetilde{V}_{q}(\vec{b})-\widetilde{V}_{q}(\vec{b}+\alpha \vec{\rho})\right|^{2} .
$$

If one assumes that the quark interacts via one-gluon exchange with the target and expands Eq. A.22 to first order in $(\alpha \rho)^{2}$, keeping only terms of order $\alpha_{s}$, then the dipole cross section is proportional to the target gluon density

We can also express the transverse momentum distribution of DY pairs in terms of the dipole cross section [5]. The differential cross section is given by the Fourier integral

$$
\begin{aligned}
\frac{d^{3} \sigma_{T, L}\left(q N \rightarrow \gamma^{*} X\right)}{d \ln \alpha d^{2} q_{\perp}} & =\frac{1}{(2 \pi)^{2}} \int d^{2} \rho_{1} d^{2} \rho_{2} \mathrm{e}^{\mathrm{i} \vec{q}_{\perp} \cdot\left(\vec{\rho}_{1}-\vec{\rho}_{2}\right)} \Psi_{\gamma^{*} q}^{* T, L}\left(\alpha, \vec{\rho}_{1}\right) \Psi_{\gamma^{*} q}^{T, L}\left(\alpha, \vec{\rho}_{2}\right) \\
& \times \frac{1}{2}\left\{\sigma_{q \bar{q}}^{N}\left(\alpha \rho_{1}\right)+\sigma_{q \bar{q}}^{N}\left(\alpha \rho_{2}\right)-\sigma_{q \bar{q}}^{N}\left(\alpha\left(\vec{\rho}_{1}-\vec{\rho}_{2}\right)\right)\right\} .
\end{aligned}
$$

To derive this expression, one performs the integration over $\vec{p}_{f \perp}$ in Eq. (A.17) and observes that Eq. (A.17) has a real value. This allows one to symmetrize the integrand with respect to $\vec{\rho}_{1}$ and $\vec{\rho}_{2}$. The functions $V_{q}$ combine then to the dipole cross sections in the second line of Eq. A.23). After integrating Eq. (A.23) over the transverse momentum $q_{\perp}$ of the photon, one obviously recovers Eq. (A.21).

\section{References}

[1] S. D. Drell and T. Yan, Phys. Rev. Lett. 25, 316 (1970) [Erratum-ibid. 25, 902 (1970)].

[2] D. M. Alde et al., Phys. Rev. Lett. 64, 2479 (1990).

[3] B. Z. Kopeliovich, proc. of the workshop Hirschegg '95: Dynamical Properties of Hadrons in Nuclear Matter, Hirschegg January 16-21, 1995, ed. by H. Feldmeyer and W. Nörenberg, Darmstadt, 1995, p. 102 (hep-ph/9609385);

[4] S. J. Brodsky, A. Hebecker and E. Quack, Phys. Rev. D 55, 2584 (1997) [hep$\mathrm{ph} / 9609384$.

[5] B. Z. Kopeliovich, A. Schäfer and A. V. Tarasov, Phys. Rev. C 59, 1609 (1999), extended version in hep-ph/9808378.

[6] J. Raufeisen, Ph.D. thesis Heidelberg 2000, hep-ph/0009358.

[7] B. Z. Kopeliovich, J. Raufeisen and A. V. Tarasov, hep-ph/0104155.

[8] M. B. Johnson, et al. [FNAL E772 Collab.], Phys. Rev. Lett. 86, 4483 (2001) [hepex/0010051; M. B. Johnson, et al., Phys. Rev. C 65, 025203 (2002) hep-ph/0105195; J. M. Moss, et al., hep-ex/0109014. 
[9] B. Z. Kopeliovich, J. Raufeisen, A. V. Tarasov and M. B. Johnson, hep-ph/0110221.

[10] B. Z. Kopeliovich, J. Raufeisen and A. V. Tarasov, Phys. Lett. B 503, 91 (2001) hepph/0012035].

[11] P. L. McGaughey, J. M. Moss and J. C. Peng, Ann. Rev. Nucl. Part. Sci. 49, 217 (1999) hep-ph/9905409].

[12] J. C. Collins, D. E. Soper and G. Sterman, Nucl. Phys. B 250, 199 (1985); J. w. Qiu and X. f. Zhang, Phys. Rev. Lett. 86, 2724 (2001) hep-ph/0012058; Phys. Rev. D 63, 114011 (2001) hep-ph/0012348.

[13] G. Altarelli, G. Parisi and R. Petronzio, Phys. Lett. B 76, 351 (1978); Phys. Lett. B 76, 356 (1978).

[14] K. J. Eskola, V. J. Kolhinen, P. V. Ruuskanen and R. L. Thews, hep-ph/0108093.

[15] J. Hüfner, Y. P. Ivanov, B. Z. Kopeliovich and J. Raufeisen, Eur. Phys. J. A 7, 391 (2000) nucl-th/9911064.

[16] J. D. Bjorken and J. B. Kogut, Phys. Rev. D 8, 1341 (1973).

[17] H. Plothow-Besch, Int. J. Mod. Phys. A 10, 2901 (1995); "PDFLIB: Proton, Pion and Photon Parton Density Functions, Parton Density Functions of the Nucleus and $\alpha_{s}$ Calculations", User's Manual - Version 8.04, W5051 PDFLIB, 2000.04.17, CERN-PPE.

[18] K. Golec-Biernat and M. Wüsthoff, Phys. Rev. D 59, 014017 (1999) hep-ph/9807513; Phys. Rev. D 60, 114023 (1999) hep-ph/9903358.

[19] A. B. Zamolodchikov, B. Z. Kopeliovich and L. I. Lapidus, JETP Lett. 33, 595 (1981) [Pisma Zh. Eksp. Teor. Fiz. 33, 612 (1981)]; G. Bertsch, S. J. Brodsky, A. S. Goldhaber and J. F. Gunion, Phys. Rev. Lett. 47, 297 (1981); S. J. Brodsky and A. H. Mueller, Phys. Lett. B 206, 685 (1988).

[20] G. Sterman et al. [CTEQ Collaboration], Rev. Mod. Phys. 67, 157 (1995).

[21] E. A. Hawker et al. [FNAL E866/NuSea Collaboration], Phys. Rev. Lett. 80, 3715 (1998) hep-ex/9803011.

[22] H. L. Lai et al. [CTEQ Collaboration], Eur. Phys. J. C 12, 375 (2000) hep-ph/9903282].

[23] P. L. McGaughey et al. [E772 Collaboration], Phys. Rev. D 50, 3038 (1994) [Erratumibid. D 60, 119903 (1994)].

[24] M. Glück, E. Reya and A. Vogt, Eur. Phys. J. C 5, 461 (1998) hep-ph/9806404.

[25] J. C. Webb et al. [E866/NuSea Collaboration], proceedings of the Tth Conference on Intersections of Particle and Nuclear Physics, Z. Parsa and W. J. Marciano eds., AIP Conference Proc., vol. 549, Melville, NY, USA, 2000. 
[26] B. Blättel, G. Baym, L. L. Frankfurt and M. Strikman, Phys. Rev. Lett. 70, 896 (1993); L. Frankfurt, A. Radyushkin and M. Strikman, Phys. Rev. D 55, 98 (1997) hep-ph/9610274.

[27] S. D. Drell and T. M. Yan, Phys. Rev. Lett. 24, 181 (1970); G. B. West, Phys. Rev. Lett. 24, 1206 (1970); S. J. Brodsky and G. R. Farrar, Phys. Rev. Lett. 31, 1153 (1973).

[28] B. Z. Kopeliovich, J. Nemchik, A. Schäfer and A. V. Tarasov, hep-ph/0201010.

[29] M. A. Betemps, M. B. Ducati and M. V. Machado, hep-ph/0111473, hep-ph/0206218.

[30] T. Affolder et al. [CDF Collaboration], Phys. Rev. D 63, 011101 (2001) hepex/0006025]; F. Abe et al. [CDF Collaboration], Phys. Rev. D 49, 1 (1994); Phys. Rev. Lett. 67, 2418 (1991).

[31] A. D. Martin, R. G. Roberts, W. J. Stirling and R. S. Thorne, Eur. Phys. J. C 4, 463 (1998) hep-ph/9803445.

[32] D. Antreasyan et al., Phys. Rev. Lett. 48, 302 (1982).

[33] W. Buchmüller, M. F. McDermott and A. Hebecker, Nucl. Phys. B 487, 283 (1997) [Erratum-ibid. B 500, 621 (1997)] [hep-ph/9607290]; W. Buchmüller and A. Hebecker, Nucl. Phys. B 476, 203 (1996) hep-ph/9512329.

[34] S. J. Brodsky, H. C. Pauli and S. S. Pinsky, Phys. Rept. 301, 299 (1998) hep$\mathrm{ph} / 9705477$. 